\title{
SimplePHOX, a Readily Available Chiral Ligand System for Iridium-Catalyzed Asymmetric Hydrogenation
}

\author{
Sebastian P. Smidt, Frederik Menges, and Andreas Pfaltz* \\ Department of Chemistry, University of Basel, St. Johanns-Ring 19, \\ CH-4056 Basel, Switzerland, e-mail: andreas.pfaltz@unibas.ch
}

\section{- Supporting Information -}

NMR spectra were recorded on Bruker Avance 400 and $500 \mathrm{MHz}$ NMR spectrometers. When necessary, the signals were assigned by APT, DEPT, COSY, HMQC, HMBC, and NOESY measurements. Dynamic phenomena were detected in a temperature range between $190 \mathrm{~K}$ and $305 \mathrm{~K}$, when needed. Reference for ${ }^{31} \mathrm{P}$ NMR: $(\mathrm{PhO})_{3} \mathrm{P}=\mathrm{O}\left(58 \%\right.$ solution in $\left.\mathrm{CDCl}_{3}\right) \equiv-18 \mathrm{ppm}$.

IR spectra were recorded on a Perkin Elmer 1600 series FTIR spectrometer. Spectra of liquids were measured neat as thin films between two sodium chloride plates, those of solids as KBr disks. EI and FAB mass spectra were carried out on mass spectrometers VG70-250 (EI) and Finnigan MAT 312 (FAB). The ions were generated by electron ionization (EI, $70 \mathrm{eV}$ ) or bombardment with fast xenon atoms (FAB), using of 3-nitrobenzyl alcohol (3-NBA) as matrix and sometimes potassium chloride as additive in the latter case. The data are given in mass units per charge $(\mathrm{m} / \mathrm{e})$, and the intensities of the signals are indicated in percent of the basis ion. ESI (electrospray ionization) mass spectra were measured with a Finnigan MAT LCQ octapole mass spectrometer. The elemental analyses were carried out at the Department of Chemistry at the University of Basel on Leco CHN-900 (C-, H-, N-detection) and Leco RO-478 (O-detection) analyzers. The data are indicated in mass percents. For gas chromatographic analysis, Carlo Erba HRGC Mega2 Series MFC 800 chromatographs were used. Achiral separations were mostly performed on the column Restek Rtx-1701, $0.25 \mu \mathrm{m}, 30 \mathrm{~m}, 60 \mathrm{kPa} \mathrm{He}$ or $\mathrm{H}_{2}$, chiral separations on several $\beta$ - or $\gamma$-CD modified capillary columns. For HPLC analysis, Shimadzu systems with a SCL-10A System Controller, CTO-10AC column oven, LC10-AD pump system, DGU-14A degasser, and a SPDM10A Diode Array Detector or a UV-vis detector (220 nm and $254 \mathrm{~nm}$ ) were used. Chiral columns Chiracel OD-H, OB-H, OJ and Chiralpak AD from Daicel Chemical Industries Ltd. were used (250 $\mathrm{mm} \times 4.6 \mathrm{~mm}$ ) at a flow of $0.5 \mathrm{~mL} \mathrm{~min}^{-1}$ heptane/2-propanol mixtures at temperatures between $20^{\circ} \mathrm{C}$ and $40^{\circ} \mathrm{C}$. Optical rotations were measured in a Perkin Elmer Polarimeter 341, sodium 
lamp, 1 dm cuvette lengths, c in g/100 mL. Melting points were measured with a Büchi 535 melting point apparatus and are not corrected.

For thin-layer chromatography (TLC), Macherey-Nagel Polygram plates SIL G/UV254, $0.2 \mathrm{~mm}$ silica with fluorescence indicator, were used. Column chromatographic separations were performed on silica 60 (particle size 40-63 $\mu \mathrm{m}, 230-400$ mesh) purchased at Merck-Schuchardt (now VWR), or Uetikon Chemie, accelerated by pressurized air or nitrogen (flash chromatography). ${ }^{[1]}$ High-pressure hydrogenations (up to $100 \mathrm{bar}$ ) were carried out in $60 \mathrm{~mL}$ autoclaves of Premex AG, Lengnau, Switzerland. Hydrogen gas used in all experiments was purchased at Carbagas Switzerland at quality 45 (99.995\%).

Reactions with air- or moisture-sensitive compounds were performed under an argon atmosphere using standard Schlenk techniques, or in a atmosphere of purified nitrogen in a $\mathrm{M}$. Braun glove box $\left(\mathrm{H}_{2} \mathrm{O}<1\right.$ ppm, $\mathrm{O}_{2}<2$ ppm). Glassware was oven-dried and then flame-dried under vacuum prior to use.

Diethyl ether, tetrahydrofuran, and toluene were dried over sodium/benzophenone, pentane over sodium-potassium alloy/benzophenone, dichloromethane over $\mathrm{CaH}_{2}$ and freshly distilled under a stream of nitrogen prior to use. ${ }^{[2]}$ Other solvents were purchased dry at Fluka or Aldrich in septum sealed bottles and kept in an inert atmosphere over molecular sieves. Kinetic investigations were performed in dry dichloromethane from Fluka. When needed, solvents were degassed prior to use by three freeze-pump-thaw cycles. Diethylamine, di(isopropyl)amine, and triethylamine were dried over $\mathrm{CaH}_{2}$ and distilled under argon. Phosphorus trichloride was refluxed overnight under argon to remove hydrogen chloride and subsequently distilled under argon.

(E)-1,2-diphenyl-1-propene (13) was purchased from Lancaster and used without further purification. (E)-2-methyl-3-phenyl-prop-2-enol (19) was purchased from Aldrich and purified by distillation. (E)-ethyl 3-phenyl-but-2-enoate (18) is commercially available from Aldrich and was used without further purification. (E)-2-(4-methoxyphenyl)-2-butene (14), (Z)-2-(4methoxyphenyl)-2-butene (15), 6-methoxy-1-methyl-3,4-dihydronaphthalene (16), and 2-(4methoxyphenyl)-1-butene (17) were prepared and their hydrogenation products were analyzed following literature procedures. ${ }^{[3]}(-)-(S)-2-(4-t e r t-b u t y l-4,5-d i h y d r o-o x a z o l-2-y l)-p r o p a n-2-o l ~(4){ }^{[4]}$, (-)-(S)-2-(4-isopropyl-4,5-dihydro-oxazol-2-yl)-propan-2-ol $\quad(5)^{[5]} \quad$ and $\quad$ chloro-di(2methylphenyl)phosphine ${ }^{[6]}$ were prepared according to literature procedures. Sodium tetrakis[3,5bis(trifluoromethyl)phenyl]borate $\left(\mathrm{NaBAr}_{\mathrm{F}}\right)$ was prepared according to literature procedures. ${ }^{[7]}$ 


\section{General conditions for catalytic hydrogenations at elevated pressure}

A solution of $0.1 \mathrm{mmol}$ substrate and $1 \mathrm{~mol} \%$ iridium pre-catalyst in $0.5 \mathrm{~mL}$ dry dichloromethane (Fluka crown-cap, over molecular sieves) was stirred in a high-pressure autoclave under 50 bar hydrogen gas for two hours. Stirring was done with magnetic stir bars at $700 \mathrm{~min}^{-1}$. All reactions were performed at room temperature. Either one reaction was run at a time in a $35 \mathrm{~mL}$ glass-insert, or four reactions were run in one autoclave in $2 \mathrm{~mL}$ glass vials that were individually stirred. Test reactions showed consistent results for both methods and no gas-phase diffusion of the more volatile substrates from one vial to another was observed. Work-up consisted in evaporating the solvent in a stream of nitrogen or argon and extraction of the hydrogenation product in $3 \mathrm{~mL}$ heptanes (HPLC quality). After filtration through a syringe filter $(0.2 \mu \mathrm{m}$, Macherey-Nagel CHROMAFIL Type O20/15, for organic solvents), these solutions were directly used for GC and HPLC analyses.

\section{General conditions for catalytic hydrogenations at ambient pressure}

A solution of $0.1-0.2 \mathrm{mmol}$ substrate and $1 \mathrm{~mol} \%$ iridium pre-catalyst in $1-2 \mathrm{ml}$ dry dichloromethane (Fluka crown-cap, over molecular sieves) was prepared under inert conditions in a $15 \mathrm{ml}$ Schlenk flask. The substrates were not dried or degassed prior to use. The reaction mixture was stirred for 30 min with slow bubbling of hydrogen gas through the solution, introduced through a stainless-steel needle. The temperature was either $25^{\circ} \mathrm{C}$ or $0^{\circ} \mathrm{C}$ and kept constant by a water or ice bath. Work-up and analyses were performed as described for the high pressure reactions.

\section{General Procedure A:}

\section{Synthesis of iridium P,N 1,5-cyclooctadiene $\mathrm{BAr}_{\mathrm{F}}{ }^{-}$complexes}

Under argon, e. g. $0.24 \mathrm{mmol} \mathrm{P}, \mathrm{N}$ ligand was dissolved in $5 \mathrm{~mL}$ dry $\mathrm{CH}_{2} \mathrm{Cl}_{2}$ and while stirring, 0.5 equiv $[\operatorname{IrCl}(1,5-\mathrm{cod})]_{2}$ was added as a solid. The red solution was heated for $2 \mathrm{~h}$ to $48{ }^{\circ} \mathrm{C}$ in a closed vial. After cooling to room temperature, 1.3 equiv $\mathrm{NaBAr}_{\mathrm{F}}$ was added, resulting in a lighter color. After $5 \mathrm{~min}, 5 \mathrm{~mL}$ water was added and the biphasic mixture was vigorously stirred for 15 min. The workup was then done in air. Phase separation was followed with extraction of the organic layer with water (two times) and drying by filtration over $\mathrm{MgSO}_{4}$. Column chromatography on silica with $\mathrm{CH}_{2} \mathrm{Cl}_{2}$ or mixtures of $\mathrm{CH}_{2} \mathrm{Cl}_{2}$ and EtOAc afforded the pure complexes that were generally recrystallized from $\mathrm{Et}_{2} \mathrm{O}$ /pentane to yield orange crystals in

up to $85 \%$ yield. 


\section{(-)-(S)-4-tert-butyl-2-(1-diphenylphosphinite-1-methyl-ethyl)-4,5-dihydro-oxazole (6)}

$100 \mathrm{mg}$ (0.540 mmol) 2-(4-tert-butyl-4,5-dihydro-oxazol-2-yl)-propan-2-ol (4) was dissolved in $10 \mathrm{~mL}$ dry pentane and $5 \mathrm{~mL}$ dry diethyl ether and $752 \mu \mathrm{l}$ (5.4 mmol, 10 equiv) triethylamine was added. At $-78{ }^{\circ} \mathrm{C}, 370 \mu \mathrm{l}\left(0.594 \mathrm{mmol}, 1.1\right.$ equiv) ${ }^{n} \mathrm{BuLi}$ solution ( $\sim 1.6 \mathrm{M}$ in hexanes) was added dropwise. After $15 \mathrm{~min}$. stirring at this temperature, the mixture was stirred at $0{ }^{\circ} \mathrm{C}$ for $30 \mathrm{~min}$, and $109 \mu \mathrm{l}$ (131 mg, 0.594 mmol, 1.1 equiv) chlorodiphenylphosphine was added dropwise. The mixture was allowed to warm to room temperature overnight. Column chromatography under argon on silica with pentane $: \mathrm{Et}_{2} \mathrm{O}=9: 1$ afforded a colorless oil that solidified to a sticky solid upon lyophilization with cyclohexane. Yield: $72 \mathrm{mg}$ (0.195 mmol, 36\%).

$\mathrm{C}_{22} \mathrm{H}_{28} \mathrm{NO}_{2} \mathrm{P}$ (369.44);

${ }^{1} \mathbf{H}$ NMR (400.1 MHz, $\mathrm{CDCl}_{3}, 300 \mathrm{~K}$ ): $\delta=0.85$ (s, $9 \mathrm{H},{ }^{t} \mathrm{Bu} \mathrm{CH}_{3}$ ), 1.63 (s, $3 \mathrm{H}, \mathrm{P}-\mathrm{O}-\mathrm{C}-\mathrm{CH}_{3}$ ), 1.65 (s, $\left.3 \mathrm{H}, \mathrm{P}-\mathrm{O}-\mathrm{C}-\mathrm{CH}_{3}\right), 3.83$ (dd, ${ }^{3} \mathrm{~J}(\mathrm{H}, \mathrm{H})=9.9 \mathrm{~Hz}, 7.6 \mathrm{~Hz}, 1 \mathrm{H}$, oxaz $\mathrm{CH}$ ), 4.02 (mc, $2 \mathrm{H}$, oxaz $\mathrm{CH}_{2}$ ), 7.35-7.27 (m, $6 \mathrm{H}$, meta and para arom. $\mathrm{CH}$ ), 7.53-7.47 (m, ortho arom. $\mathrm{CH}$ );

${ }^{31} \mathbf{P}\left\{{ }^{1} \mathbf{H}\right\}$ NMR (162.0 MHz, $\left.\mathrm{CDCl}_{3}, 300 \mathrm{~K}\right): \delta=94.9$ (s);

IR (KBr): $\widetilde{v}=$ 3397vs,br, 3059m, 2962vs, 2870s, 2722w, 1972vw, 1922vw, 1734m, 1684vs, 1648m, 1592m, 1523s, 1475m, 1439s, 1397w, 1380m, 1365m, 1262w, 1214vs, 1129vs, 1111vs, 1058m, 1009s, 979vs, 934m, 858m, 755m, 729s, 698s, 587m, 538s cm ${ }^{-1}$;

+FABMS, 3-NBA, m/e: 307 ([M - $\left.\left.\mathrm{C}_{6} \mathrm{H}_{6}+\mathrm{O}\right]^{+}, 100 \%\right)$;

$[\alpha]_{D}^{20}=-52.8\left(\mathrm{c}=0.5, \mathrm{CHCl}_{3}\right)$;

\section{(-)-(S)-4-tert-butyl-2-\{1-di(2'-methylphenyl)phosphinite-1-methyl-ethyl\}-4,5-dihydro-oxazole (7)}

$500 \mathrm{mg}$ (2.7 mmol) 2-(4-tert-butyl-4,5-dihydro-oxazol-2-yl)-propan-2-ol (4) was dissolved in $50 \mathrm{~mL}$ dry pentane and at $-78{ }^{\circ} \mathrm{C}, 2.2 \mathrm{~mL}$ (3.51 mmol, 1.3 equiv) ${ }^{n}$ BuLi solution ( 1.6 $\mathrm{M}$ in hexanes) was added dropwise. After 10 min., $0.82 \mathrm{~mL}$ (5.4 mmol, 2 equiv) TMEDA was added and a pink colored suspension was obtained. After $1.5 \mathrm{~h}$ stirring at $-78{ }^{\circ} \mathrm{C}$, the mixture was allowed to warm to room temperature over $1 \mathrm{~h}$. To this white suspension, a solution of $873 \mathrm{mg}$ (3.51 mmol, $1.3 \mathrm{equiv}$ ) di(2methylphenyl)-chloro-phosphine in $10 \mathrm{~mL}$ pentane was added dropwise at $0^{\circ} \mathrm{C}$. The mixture was allowed to warm to room temperature overnight and the solvent volume was reduced to $2 \mathrm{~mL}$. This suspension was transferred directly on a column prepared under argon (silica, pentane $\left.: \mathrm{Et}_{2} \mathrm{O}: \mathrm{NEt}_{3}=95: 5: 1\right)$. After chromatography, a colorless oil was obtained. Yield: $536 \mathrm{mg}$ (1.35 mmol, 50\%).

$\mathrm{C}_{24} \mathrm{H}_{32} \mathrm{NO}_{2} \mathrm{P}$ (397.49); 
${ }^{1} \mathbf{H}$ NMR (400.1 MHz, $\left.\mathrm{C}_{6} \mathrm{D}_{6}, 300 \mathrm{~K}\right): \delta=0.76\left(\mathrm{~s}, 9 \mathrm{H},{ }^{t} \mathrm{Bu} \mathrm{CH}_{3}\right.$ ), 1.69 (s, $\left.3 \mathrm{H}, \mathrm{P}-\mathrm{O}-\mathrm{C}-\mathrm{CH}_{3}\right), 1.72$ (s, $3 \mathrm{H}, \mathrm{P}-\mathrm{O}-\mathrm{C}-\mathrm{CH}_{3}$ ), 2.37 (s, $3 \mathrm{H},{ }^{\circ} \mathrm{Tol} \mathrm{CH}_{3}$ ), 2.42 (s, $3 \mathrm{H},{ }^{\circ} \mathrm{Tol} \mathrm{CH}_{3}$ ), $3.60\left(\mathrm{dd},{ }^{3} \mathrm{~J}(\mathrm{H}, \mathrm{H}) \approx 8 \mathrm{~Hz}, 8 \mathrm{~Hz}\right.$, $1 \mathrm{H}$, oxaz $\mathrm{CH}$ ), 3.65 (“t”, $J(\mathrm{H}, \mathrm{H}) \approx 8 \mathrm{~Hz}, 1 \mathrm{H}$, oxaz $\mathrm{CH}_{2}$ ), 3.68 (“t”, $J(\mathrm{H}, \mathrm{H}) \approx 8 \mathrm{~Hz}, 1 \mathrm{H}$, oxaz $\mathrm{CH}_{2}$ ), 6.90-6.94 (m, 2 H, arom. CH), 7.01-7.15 (m, 4 H, arom. CH), 7.95 (mc, 2 H, ortho arom. CH);

${ }^{31} \mathbf{P}\left\{{ }^{1} \mathbf{H}\right\}$ NMR (162.0 MHz, $\left.{ }_{6} \mathrm{D}_{6}, 300 \mathrm{~K}\right): \delta=81.2(\mathrm{~s})$;

${ }^{13} \mathbf{C}\left\{{ }^{1} \mathbf{H}\right\}$ NMR (100.6 MHz, $\left.\mathrm{C}_{6} \mathrm{D}_{6}, 300 \mathrm{~K}\right): \delta=20.7\left(\mathrm{~d},{ }^{3} \mathrm{~J}(\mathrm{P}, \mathrm{C})=3.8 \mathrm{~Hz}\right.$, oxaz-CCH$\left.{ }_{3}\right), 20.9(\mathrm{~d}$, ${ }^{3} J(\mathrm{P}, \mathrm{C})=4.2 \mathrm{~Hz}$, oxaz-CCH 3$), 26.0\left(\mathrm{~s},{ }^{t} \mathrm{Bu} \mathrm{CH}_{3}\right), 27.6\left(\mathrm{~d},{ }^{3} \mathrm{~J}(\mathrm{P}, \mathrm{C})=8.8 \mathrm{~Hz},{ }^{\circ} \mathrm{Tol}^{-} \mathrm{CH}_{3}\right), 27.7(\mathrm{~d}$,

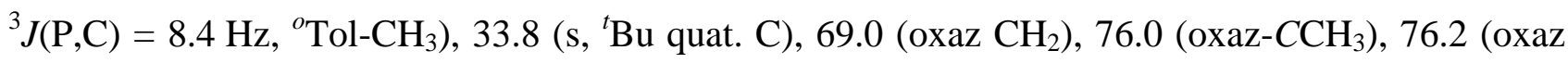
$\mathrm{CH}), 126.2$ (“t”, ${ }^{3} J(\mathrm{P}, \mathrm{C})=3.1 \mathrm{~Hz}, 2 \mathrm{C}$, meta arom. C), 129.3, 129.4, $130.2(\mathrm{~d}, J(\mathrm{P}, \mathrm{C})=3.4 \mathrm{~Hz})$, $130.3(\mathrm{~d}, J(\mathrm{P}, \mathrm{C})=3.4 \mathrm{~Hz}), 131.8(\mathrm{~d}, J(\mathrm{P}, \mathrm{C})=8.4 \mathrm{~Hz}), 132.2(\mathrm{~d}, J(\mathrm{P}, \mathrm{C})=8.8 \mathrm{~Hz}), 141.0$ (superimposed d, 4 C, quat. arom. C), 168.8 (oxaz quat. C);

IR (neat, on NaCl): $\tilde{v}=3055 \mathrm{~m}, 2957 \mathrm{vs}, 2904 \mathrm{~m}, 2868 \mathrm{~m}, 1920 \mathrm{vw}, 1730 \mathrm{w}, 1666 \mathrm{vs}, 1590 \mathrm{w}$, 1566vw, 1508vw, 1467s, 1454s, 1394w, 1380m, 1364m, 1271m, 1208m, 1157vs, 1131vs, 1068w, 1031w, 979s, 958s, 921m, 877s, 800w, 749vs, 720m, 673w cm ${ }^{-1}$;

+EI MS, $70 \mathrm{eV}, \mathrm{m} / \mathrm{e}: 168\left(\left[\mathrm{M}-\mathrm{OP}^{\mathrm{o}} \mathrm{Tol}_{2}\right]^{+}, 100 \%\right), 215\left(\left[\mathrm{M}-{ }^{\circ} \mathrm{Tol}_{2}\right]^{+}, 29 \%\right), 229\left(\left[\mathrm{OP}^{\circ} \mathrm{Tol}_{2}\right]^{+}\right.$, $88 \%), 397\left(\mathrm{M}^{+}, 3 \%\right)$;

$[\alpha]_{D}^{20}=-38.2\left(\mathrm{c}=0.7, \mathrm{CHCl}_{3}\right)$;

TLC: $R_{f}=0.32$ (pentane $: \mathrm{Et}_{2} \mathrm{O}: \mathrm{NEt}_{3}=95: 5: 1$ on silica).

\section{(S)-4-isopropyl-2-(1-diphenylphosphinite-1-methyl-ethyl)-4,5-dihydro-oxazole (8)}

$300 \mathrm{mg}$ (1.75 mmol) 2-(4-isopropyl-4,5-dihydro-oxazol-2-yl)-propan-2-ol (5) was dissolved in $20 \mathrm{~mL}$ dry pentane and $340 \mu \mathrm{L}$ ( $2.28 \mathrm{mmol}, 1.3$ equiv) TMEDA was added. At $-78{ }^{\circ} \mathrm{C}, 1.42 \mathrm{~mL}$ (2.28 mmol, 1.3 equiv) ${ }^{n}$ BuLi solution ( 1.6 M in hexanes) was added dropwise. After $15 \mathrm{~min}$. stirring at this temperature, the mixture was stirred at $0{ }^{\circ} \mathrm{C}$ for $60 \mathrm{~min}$, and $420 \mu \mathrm{L}(2.28 \mathrm{mmol}$, 1.3 equiv) chlorodiphenylphosphine was added dropwise. The mixture was allowed to warm to room temperature overnight. Column chromatography under argon on silica with pentane : $\mathrm{Et}_{2} \mathrm{O}=$ $8: 1$ afforded a colorless oil. Yield: $382 \mathrm{mg}$ (1.08 mmol, 62\%).

$\mathrm{C}_{21} \mathrm{H}_{26} \mathrm{NO}_{2} \mathrm{P}$ (355.41);

${ }^{1} \mathbf{H}$ NMR (400.1 MHz, CDCl $\left.3,300 \mathrm{~K}\right): \delta=0.82\left(\mathrm{~d},{ }^{3} \mathrm{~J}(\mathrm{H}, \mathrm{H})=6.6 \mathrm{~Hz}, 3 \mathrm{H},{ }^{i} \mathrm{Pr} \mathrm{CH}_{3}\right), 0.93\left(\mathrm{~d},{ }^{3} J(\mathrm{H}, \mathrm{H})\right.$ $=6.8 \mathrm{~Hz}, 3 \mathrm{H},{ }^{i} \mathrm{Pr} \mathrm{CH}_{3}$ ), 1.64 (s, $3 \mathrm{H}, \mathrm{P}-\mathrm{O}-\mathrm{C}-\mathrm{CH}_{3}$ ), 1.66 (s, $3 \mathrm{H}, \mathrm{P}-\mathrm{O}-\mathrm{C}-\mathrm{CH}_{3}$ ), 1.72 (sep, $J=6.6 \mathrm{~Hz}$, $1 \mathrm{H},{ }^{i} \mathrm{Pr} \mathrm{CH}$ ), 3.89 and 4.07 (m, together $3 \mathrm{H}$, oxaz $\mathrm{CH}$ and oxaz $\left.\mathrm{CH}_{2}\right), 7.29-7.51(\mathrm{~m}, 10 \mathrm{H}$, arom. CH) ppm. 
${ }^{31} \mathbf{P}\left\{{ }^{1} \mathbf{H}\right\}$ NMR $\left(162.0 \mathrm{MHz}, \mathrm{CDCl}_{3}, 300 \mathrm{~K}\right): \delta=92.4 \mathrm{ppm}$.

${ }^{13} \mathbf{C}\left\{{ }^{1} \mathbf{H}\right\}$ NMR (100.6 MHz, $\left.\mathrm{CDCl}_{3}, 300 \mathrm{~K}\right): \delta=18.2\left({ }^{i} \operatorname{Pr} \mathrm{CH}_{3}\right), 19.1\left({ }^{i} \mathrm{Pr} \mathrm{CH}_{3}\right), 27.7\left(\mathrm{~d},{ }^{3} J(\mathrm{P}, \mathrm{C})=\right.$ $\left.8.8 \mathrm{~Hz}, \mathrm{P}-\mathrm{O}-\mathrm{C}-\mathrm{CH}_{3}\right), 27.9$ (d, $\left.{ }^{3} \mathrm{~J}(\mathrm{P}, \mathrm{C})=8.8 \mathrm{~Hz}, \mathrm{P}-\mathrm{O}-\mathrm{C}-\mathrm{CH}_{3}\right), 32.7$ ( ${ }^{\mathrm{P} r \mathrm{CH}),} 70.4\left(\mathrm{oxaz} \mathrm{CH}_{2}\right), 72.3$ (oxaz CH), 76.0 (d, $\left.{ }^{2} J(\mathrm{P}, \mathrm{C})=14.9 \mathrm{~Hz}, \mathrm{P}-\mathrm{O}-\mathrm{C}-\mathrm{CH}_{3}\right), 128.5$ (d, J(P,C) = 6.9 Hz, arom. CH), 129.2 (d, $J(\mathrm{P}, \mathrm{C})=3.4 \mathrm{~Hz}$, arom. CH), $130.6(\mathrm{~d}, J(\mathrm{P}, \mathrm{C})=11.0 \mathrm{~Hz}$, arom. CH), $130.8(\mathrm{~d}, J(\mathrm{P}, \mathrm{C})=10.7 \mathrm{~Hz}$, arom. CH), 143.3 (d, J(P,C) = $1.9 \mathrm{~Hz}$, arom. C), 143.4 (d, J(P,C) = $1.9 \mathrm{~Hz}$, arom. C), $169.2(\mathrm{C}=\mathrm{N})$ ppm.

TLC: $R_{f}=0.20$ (pentane $: \mathrm{Et}_{2} \mathrm{O}=8: 1$ on silica).

(S)-4-isopropyl-2-\{1-di(2'-methylphenyl)phosphinite-1-methyl-ethyl\}-4,5-dihydro-oxazole (9) $300 \mathrm{mg}$ (1.75 mmol) 2-(4-isopropyl-4,5-dihydro-oxazol-2-yl)-propan-2-ol (5) was dissolved in $20 \mathrm{~mL}$ dry pentane and $340 \mu \mathrm{L}$ (2.28 mmol, 1.3 equiv) TMEDA was added. At $-78{ }^{\circ} \mathrm{C}, 1.42 \mathrm{~mL}$ (2.28 mmol, 1.3 equiv) ${ }^{n}$ BuLi solution ( 1.6 $\mathrm{M}$ in hexanes) was added dropwise. After $15 \mathrm{~min}$. stirring at this temperature, the mixture was stirred at $0{ }^{\circ} \mathrm{C}$ for $60 \mathrm{~min}$, and $618 \mathrm{mg}(2.28 \mathrm{mmol}$, 1.3 equiv) di(2-methylphenyl)-chloro-phosphine in $5 \mathrm{~mL}$ pentane was added dropwise. The mixture was allowed to warm to room temperature overnight. Column chromatography under argon on silica with pentane : $\mathrm{Et}_{2} \mathrm{O}=8: 1$ afforded a colorless oil. Yield: $194 \mathrm{mg}$ (0.51 mmol, 29\%).

$\mathrm{C}_{23} \mathrm{H}_{30} \mathrm{NO}_{2} \mathrm{P}$ (383.49);

${ }^{1}$ H NMR (400.1 MHz, $\left.\mathrm{CDCl}_{3}, 300 \mathrm{~K}\right): \delta=0.79\left(\mathrm{~d},{ }^{3} \mathrm{~J}(\mathrm{H}, \mathrm{H})=6.6 \mathrm{~Hz}, 3 \mathrm{H},{ }^{i} \mathrm{Pr} \mathrm{CH}_{3}\right), 0.93\left(\mathrm{~d},{ }^{3} J(\mathrm{H}, \mathrm{H})\right.$ $=6.8 \mathrm{~Hz}, 3 \mathrm{H},{ }^{i} \mathrm{Pr} \mathrm{CH}_{3}$ ), 1.63 (s, 3H, P-O-C-CH $), 1.64$ (s, 3H, P-O-C-CH $), 1.71$ (m, 1H, ${ }^{i} \mathrm{Pr} \mathrm{CH}$ ), 2.32 (s, 3H, $\left.{ }^{\circ} \mathrm{Tol}_{-} \mathrm{CH}_{3}\right), 2.38$ (s, 3H, $\left.{ }^{\circ} \mathrm{Tol}_{-} \mathrm{CH}_{3}\right), 3.83$ (m, 2H, oxaz. $\mathrm{CH}$ and oxaz. $\left.\mathrm{CH}_{2}\right), 3.96(\mathrm{~m}, 1 \mathrm{H}$, oxaz. $\mathrm{CH}_{2}$ ), 7.06 (m, 2H, arom. $\left.\mathrm{CH}\right), 7.18$ (m, 4H, arom. $\left.\mathrm{CH}\right), 7.51$ - 7.62 (m, 2H, arom. CH) ppm.

${ }^{31} \mathbf{P}\left\{{ }^{1} \mathbf{H}\right\}$ NMR $\left(162.0 \mathrm{MHz}, \mathrm{CDCl}_{3}, 300 \mathrm{~K}\right): \delta=78.0 \mathrm{ppm}$.

TLC: $R_{f}=0.22$ (pentane $: \mathrm{Et}_{2} \mathrm{O}=8: 1$ on silica).

\section{(-)-(S)-[( $\left.\eta^{4}-1,5-c y c l o o c t a d i e n e\right)-\{$ 4-tert-butyl-2-(1-diphenylphosphinite-1-methyl-ethyl)-4,5-} dihydro-oxazole \}iridium(I)]-tetrakis[3,5-bis(trifluoromethyl)phenyl]borate (Ir-6)

Synthesis according to general procedure A using $35 \mathrm{mg}(0.095 \mathrm{mmol}) \mathbf{6}, 32 \mathrm{mg}$ (0.048 mmol, 0.5 equiv) [ $\operatorname{IrCl}(1,5-\mathrm{cod})]_{2}$, and $109 \mathrm{mg}$ (0.123 mmol, 1.3 equiv) $\mathrm{NaBAr}_{\mathrm{F}}$ in $10 \mathrm{~mL} \mathrm{CH}_{2} \mathrm{Cl}_{2}$.

Yield: $100 \mathrm{mg}$ (0.065 mmol, 69\%). Single crystals suitable for X-ray crystallography were obtained by recrystallisation from $\mathrm{Et}_{2} \mathrm{O}$ /pentane (Figure $\mathrm{S} 1$ and $\mathrm{S} 2$, p. 14).

$\mathrm{C}_{62} \mathrm{H}_{52} \mathrm{BF}_{24} \mathrm{IrNO}_{2} \mathrm{P}$ (1533.05): calcd C 48.57, H 3.42, N 0.91, O 2.09; 
found C 48.61, H 3.49, N 0.77, O 2.37;

m. p. $201^{\circ} \mathrm{C}$;

${ }^{1} \mathbf{H}$ NMR (500.1 MHz, $\left.\mathrm{CDCl}_{3}, 295 \mathrm{~K}\right): \delta=0.59$ (s, $9 \mathrm{H},{ }^{t} \mathrm{Bu} \mathrm{CH}_{3}$ ), 1.57 (mc, $1 \mathrm{H}, \mathrm{cod}-\mathrm{CH}_{2}$ ), 1.76 (s, $3 \mathrm{H}, \mathrm{P}-\mathrm{O}-\mathrm{C}-\mathrm{CH}_{3}$ ), 1.78 (mc, $1 \mathrm{H}$, cod- $\mathrm{CH}_{2}$ ), 1.97 (mc, $1 \mathrm{H}$, cod-CH ), 2.09 (mc, $1 \mathrm{H}, \mathrm{cod}-\mathrm{CH}_{2}$ ), 2.28 (mc, $1 \mathrm{H}$, cod- $\mathrm{CH}_{2}$ ), 2.35 (s, $3 \mathrm{H}, \mathrm{P}-\mathrm{O}-\mathrm{C}-\mathrm{CH}_{3}$ ), 2.37 (mc, $1 \mathrm{H}$, cod-CH ), 2.58 (mc, $2 \mathrm{H}$, cod-CH ), 2.69 (mc, $1 \mathrm{H}$, cod-CH), 3.71 (mc, $1 \mathrm{H}$, cod-CH), 3.76 (mc, $1 \mathrm{H}$, oxaz $\mathrm{CH}$ ), 4.29 (“t”, ${ }^{3} J(\mathrm{H}, \mathrm{H}) \approx$ $10 \mathrm{~Hz}, 1 \mathrm{H}$, oxaz $\mathrm{CH}_{2}$ ), 4.56 (mc, $1 \mathrm{H}$, oxaz $\mathrm{CH}_{2}$ ), 4.95 (mc, $1 \mathrm{H}$, cod-CH), 5.13 (mc, $1 \mathrm{H}$, cod-CH), 7.16 (mc, 2 H, arom. CH), 7.44 (mc, 3 H, arom. CH), 7.52 (br s, 4 H, BArF para CH), 7.54 (mc, 3 H, arom. CH), 7.73 (br “t”, 8 H, BAr F ortho $\mathrm{CH}$ ), 7.76 (mc, $2 \mathrm{H}$, arom. $\mathrm{CH}$ );

${ }^{31} \mathbf{P}\left\{{ }^{1} \mathbf{H}\right\}$ NMR (162.0 MHz, $\left.\mathrm{CDCl}_{3}, 300 \mathrm{~K}\right): \delta=99.8$ (s);

${ }^{19} \mathbf{F}\left\{{ }^{1} \mathbf{H}\right\}$ NMR (376.5 MHz, $\left.\mathrm{CDCl}_{3}, 300 \mathrm{~K}\right): \delta=-63.5$ (s);

${ }^{13} \mathrm{C}\left\{{ }^{1} \mathbf{H}\right\}$ NMR $\left(100.6 \mathrm{MHz}, \mathrm{CDCl}_{3}, 300 \mathrm{~K}\right): \delta=24.8\left(3 \mathrm{C},{ }^{t} \mathrm{Bu} \mathrm{CH}_{3}\right), 25.5\left(\mathrm{~d},{ }^{4} \mathrm{~J}(\mathrm{P}, \mathrm{C})=1.5 \mathrm{~Hz}\right.$, cod $\left.\mathrm{CH}_{2}\right), 26.0\left(\mathrm{~d},{ }^{4} J(\mathrm{P}, \mathrm{C})=6.1 \mathrm{~Hz}, \mathrm{oxaz}^{-}{ }^{t} \mathrm{Bu}\right.$ quat. C$), 28.9\left(\operatorname{cod} \mathrm{CH}_{2}\right), 32.2\left(\mathrm{~d},{ }^{2} J(\mathrm{P}, \mathrm{C})=2.3 \mathrm{~Hz}, \mathrm{oxaz}-\right.$ $\left.\mathrm{C}\left(\mathrm{CH}_{3}\right)_{2}\right), 32.7\left(\mathrm{~d},{ }^{4} \mathrm{~J}(\mathrm{P}, \mathrm{C})=3.1 \mathrm{~Hz}, \operatorname{cod} \mathrm{CH}_{2}\right), 33.5\left(2 \mathrm{C}\right.$, oxaz-C$\left.\left(\mathrm{CH}_{3}\right)_{2}\right), 36.2\left(\mathrm{~d},{ }^{4} \mathrm{~J}(\mathrm{P}, \mathrm{C})=3.8 \mathrm{~Hz}\right.$, cod- $\mathrm{CH}_{2}$ ), 60.6 (cod $\mathrm{CH}$ ), 64.5 (cod $\mathrm{CH}$ ), 72.0 (oxaz $\mathrm{CH}_{2}$ ), 74.4 (oxaz $\left.\mathrm{CH}\right), 97.4$ (d, ${ }^{2} J(\mathrm{P}, \mathrm{C})=13.0 \mathrm{~Hz}$, cod CH), $99.8\left(\mathrm{~d},{ }^{2} J(\mathrm{P}, \mathrm{C})=12.3 \mathrm{~Hz}\right.$, cod CH), $117.5\left(\mathrm{sept},{ }^{3} \mathrm{~J}(\mathrm{C}, \mathrm{F})=3.8 \mathrm{~Hz}, 4 \mathrm{C}\right.$, $\mathrm{BAr}_{\mathrm{F}}$ para CH), $124.5\left(\mathrm{q},{ }^{1} J(\mathrm{~F}, \mathrm{C})=272.5 \mathrm{~Hz}, 8 \mathrm{C}, \mathrm{BAr}_{\mathrm{F}} \mathrm{CF}_{3}\right), 128.9,129.0,129.2,129.3,129.9$ (qq, ${ }^{2} J(F, C)=31.2 \mathrm{~Hz},{ }^{3} J(B, C)=2.9 \mathrm{~Hz}, 8 \mathrm{C}, \mathrm{BAr}_{\mathrm{F}}$ quat. C ipso to $\mathrm{CF}_{3}$ ), 129.9, 130.0, 130.0 (quat. C), 131.9, 132.0 (2 C), 132.8 (d, J(P,C) = 2.3 Hz), 134.2 (quat. C), 134.0, 134.8 (br, 8 C, BAr Frtho $\mathrm{CH}), 161.7\left(\mathrm{q},{ }^{1} \mathrm{~J}(\mathrm{~B}, \mathrm{C})=49.9 \mathrm{~Hz}, 4 \mathrm{C}, \mathrm{BAr}_{\mathrm{F}}\right.$ quat. C ipso to B), 175.5 (d, ${ }^{3} \mathrm{~J}(\mathrm{P}, \mathrm{C})=6.1 \mathrm{~Hz}$, quat. C oxaz);

IR (KBr): $\tilde{v}=3441 \mathrm{br}, 3064 \mathrm{w}, 2965 \mathrm{w}, 2891 \mathrm{w}, 2841 \mathrm{vw}, 1611 \mathrm{~s}, 1485 \mathrm{w}, 1428 \mathrm{~m}, 1398 \mathrm{~m}, 1354 \mathrm{vs}$, 1276vs, 1167vs, 1129vs, 1005m, 971m, 954m, 898s, 886s, 838s, 769m, 743m, 716s, 704m, 682s, 668s, 570s, 522w, 488w, 447w cm ${ }^{-1}$;

+ESIMS, MeOH, m/e: $670.2\left(\left[\mathrm{M}-\mathrm{BAr}_{\mathrm{F}}\right]^{+}, 100 \%\right)$;

$[\alpha]_{D}^{20}=-12.5\left(\mathrm{c}=0.5, \mathrm{CHCl}_{3}\right)$.

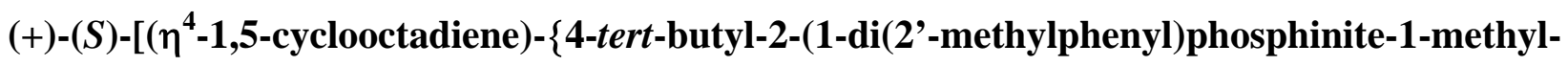
ethyl)-4,5-dihydro-oxazole \}iridium(I)]-tetrakis[3,5-bis(trifluoromethyl)phenyl]borate (Ir-7)

Synthesis according to general procedure A using $200 \mathrm{mg}(0.503 \mathrm{mmol})$ 7, $169 \mathrm{mg}(0.252 \mathrm{mmol}$, 0.5 equiv) $[\mathrm{IrCl}(1,5-\mathrm{cod})]_{2}$, and $580 \mathrm{mg}\left(0.654 \mathrm{mmol}, 1.3\right.$ equiv) $\mathrm{NaBAr}_{\mathrm{F}}$ in $20 \mathrm{~mL} \mathrm{CH}_{2} \mathrm{Cl}_{2}$. Yield: 664 mg (0.426 mmol, 85\%). 
$\mathrm{C}_{64} \mathrm{H}_{56} \mathrm{BF}_{24} \mathrm{IrNO}_{2} \mathrm{P}$ (1561.10): calcd C 49.24, H 3.62, $\mathrm{N}$ 0.90, O 2.05;

found C 49.29, H 3.61, N 0.84, O 2.77;

m. p. $198^{\circ} \mathrm{C}$;

${ }^{1} \mathbf{H}$ NMR (500.1 MHz, $\mathrm{CD}_{2} \mathrm{Cl}_{2}, 220 \mathrm{~K}$ ): $\delta=0.79$ (br s, $9 \mathrm{H},{ }^{t} \mathrm{Bu} \mathrm{CH}_{3}$ ), 1.42 (mc, $1 \mathrm{H}$, cod-CH ), 1.44

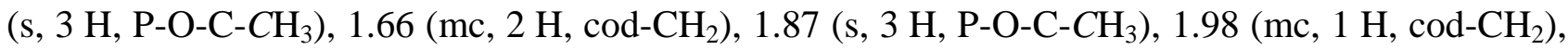
2.06 (mc, $1 \mathrm{H}$, cod-CH $\mathrm{CH}_{2}$ ), 2.09 (s, $3 \mathrm{H},{ }^{\circ}{ }^{\mathrm{Tol}}-\mathrm{CH}_{3}$ ), 2.46 (mc, $3 \mathrm{H}$, cod-CH $\mathrm{CH}_{2}, 2.46$ (mc, $1 \mathrm{H}$, cod-CH), 2.80 (s, $3 \mathrm{H},{ }^{\circ} \mathrm{Tol}-\mathrm{CH}_{3}$ ), 3.41 (mc, $1 \mathrm{H}$, cod-CH), $3.91\left(\mathrm{dd},{ }^{3} \mathrm{~J}(\mathrm{H}, \mathrm{H})=9.3 \mathrm{~Hz}, 2.7 \mathrm{~Hz}, 1 \mathrm{H}\right.$, oxaz CH), 4.31 ("t", ${ }^{3} J(\mathrm{H}, \mathrm{H}) \approx 9.5 \mathrm{~Hz}, 1 \mathrm{H}$, oxaz $\mathrm{CH}_{2}$ trans to $\left.{ }^{t} \mathrm{Bu}\right), 4.57$ (dd, ${ }^{2} J(\mathrm{H}, \mathrm{H})=9.9 \mathrm{~Hz}$, ${ }^{3} J(\mathrm{H}, \mathrm{H})=2.7 \mathrm{~Hz}, 1 \mathrm{H}$, oxaz $\mathrm{CH}_{2}$ cis to ${ }^{t} \mathrm{Bu}$ ), 4.82 (mc, $1 \mathrm{H}$, cod-CH), 5.33 (mc, $1 \mathrm{H}$, cod-CH), 6.46 (mc, $1 \mathrm{H}$, arom. $\mathrm{CH}$ ), 7.03 (mc, $1 \mathrm{H}$, arom. $\mathrm{CH}$ ), 7.32 (mc, $4 \mathrm{H}$, arom. $\mathrm{CH}$ ), 7.48 (mc, $2 \mathrm{H}$, arom. $\mathrm{CH}$ ), 7.52 (br s, 4 H, BArF para CH), 7.73 (br “t”, 8 H, BAr F $_{\text {Ftho }} \mathrm{CH}$ ), 8.36 (mc, $1 \mathrm{H}$, arom. $\mathrm{CH}$ ortho to $\mathrm{P}$ ). The signals belonging to the minor conformer (6\%) were neglected;

${ }^{31} \mathbf{P}\left\{{ }^{1} \mathbf{H}\right\}$ NMR (202.5 MHz, $\mathrm{CD}_{2} \mathrm{Cl}_{2}, 220 \mathrm{~K}$ ): two conformers, $\delta=112.2$ (s, 94\%), 100.1 (s, 6\%);

${ }^{19} \mathbf{F}\left\{{ }^{1} \mathbf{H}\right\}$ NMR (376.5 MHz, $\left.\mathrm{CD}_{2} \mathrm{Cl}_{2}, 300 \mathrm{~K}\right): \delta=-63.5(\mathrm{~s})$;

${ }^{13} \mathbf{C}\left\{{ }^{1} \mathbf{H}\right\}$ NMR (125.8 MHz, $\left.\mathrm{CD}_{2} \mathrm{Cl}_{2}, 220 \mathrm{~K}\right): \delta=20.6\left(\mathrm{~d},{ }^{3} J(\mathrm{P}, \mathrm{C})=3.4 \mathrm{~Hz},{ }^{o} \mathrm{Tol}-\mathrm{CH}_{3}\right), 22.5(\mathrm{~d}$, $\left.{ }^{3} J(\mathrm{P}, \mathrm{C})=3.4 \mathrm{~Hz},{ }^{\circ} \mathrm{Tol}-\mathrm{CH}_{3}\right), 23.6\left(\operatorname{cod} \mathrm{CH}_{2}\right), 26.4\left(\right.$ oxaz-C$\left.\left(\mathrm{CH}_{3}\right)_{2}\right), 26.7\left(\right.$ oxaz-C $\left.\left(\mathrm{CH}_{3}\right)_{2}\right), 27.7$ (d, ${ }^{4} J(\mathrm{P}, \mathrm{C})=54.2 \mathrm{~Hz}$, oxaz- ${ }^{t} \mathrm{Bu}$ quat. C), $30.1\left(\operatorname{cod~} \mathrm{CH}_{2}\right), 32.6$ (oxaz- $\left.C\left(\mathrm{CH}_{3}\right)_{2}\right), 32.7\left(\mathrm{cod}-\mathrm{CH}_{2}\right), 33.5$ (3 C, ${ }^{t} \mathrm{Bu} \mathrm{CH}$ ), 36.5 (d, ${ }^{4} \mathrm{~J}(\mathrm{P}, \mathrm{C})=3.4 \mathrm{~Hz}$, cod $\left.\mathrm{CH}_{2}\right), 61.9(\operatorname{cod} \mathrm{CH}), 70.5\left(\mathrm{oxaz} \mathrm{CH}_{2}\right), 73.4(\mathrm{oxaz}$ $\mathrm{CH}), 79.5(\operatorname{cod} \mathrm{CH}), 94.6\left(\mathrm{~d},{ }^{2} J(\mathrm{P}, \mathrm{C})=13.9 \mathrm{~Hz}, \operatorname{cod} \mathrm{CH}\right), 98.9\left(\mathrm{~d},{ }^{2} \mathrm{~J}(\mathrm{P}, \mathrm{C})=11.0 \mathrm{~Hz}, \operatorname{cod} \mathrm{CH}\right)$, 117.5 (sept, ${ }^{3} J(\mathrm{C}, \mathrm{F})=3.8 \mathrm{~Hz}, 4 \mathrm{C}, \mathrm{BAr}_{\mathrm{F}}$ para CH), 124.5 (q, ${ }^{1} J(\mathrm{~F}, \mathrm{C})=272.5 \mathrm{~Hz}, 8 \mathrm{C}, \mathrm{BAr}_{\mathrm{F}} \mathrm{CF}_{3}$ ), 124.5 (quat. C), 124.8 (d, $J(\mathrm{P}, \mathrm{C})=16.8 \mathrm{~Hz}$ ), 125.2 (quat. C), 130.3 (d, $J(\mathrm{P}, \mathrm{C})=15.8 \mathrm{~Hz}), 129.9$ (qq, ${ }^{2} J(\mathrm{~F}, \mathrm{C})=31.2 \mathrm{~Hz},{ }^{3} J(\mathrm{~B}, \mathrm{C})=2.9 \mathrm{~Hz}, 8 \mathrm{C}, \mathrm{BAr}_{\mathrm{F}}$ quat. C ipso to $\left.\mathrm{CF}_{3}\right), 131.1(\mathrm{~d}, J(\mathrm{P}, \mathrm{C})=8.2 \mathrm{~Hz})$, 131.6, 131.9 (quat. C), 132.1 (d, $J(\mathrm{P}, \mathrm{C})=6.7 \mathrm{~Hz}$, quat. C), 132.4, 134.8 (br, $8 \mathrm{C}, \mathrm{BAr}_{\mathrm{F}}$ ortho $\mathrm{CH}$ ), $139.4(\mathrm{~d}, J(\mathrm{P}, \mathrm{C})=33.1 \mathrm{~Hz}), 141.0(\mathrm{~d}, J(\mathrm{P}, \mathrm{C})=7.7 \mathrm{~Hz}), 141.6,161.7\left(\mathrm{q},{ }^{1} J(\mathrm{~B}, \mathrm{C})=49.9 \mathrm{~Hz}, 4 \mathrm{C}\right.$, $\mathrm{BAr}_{\mathrm{F}}$ quat. C ipso to $\left.\mathrm{B}\right), 172.6\left(\mathrm{~d},{ }^{3} J(\mathrm{P}, \mathrm{C})=9.6 \mathrm{~Hz}\right.$, quat. C oxaz);

IR (KBr): $\widetilde{v}=3060 \mathrm{w}, 2969 \mathrm{~m}, 1610 \mathrm{~m}, 1466 \mathrm{~m}, 1355 \mathrm{~s}, 1277 \mathrm{vs}, 1160 \mathrm{~s}, 1128 \mathrm{br}, \mathrm{vs}, 1018 \mathrm{w}, 990 \mathrm{w}$, 887w, 839w, 753w, 714w, 714m, 682m, 665m, 578w cm ${ }^{-1}$;

+FABMS, 3-NBA, m/e: $698\left(\left[\mathrm{M}-\mathrm{BAr}_{\mathrm{F}}\right]^{+}, 100 \%\right), 588\left(\left[\mathrm{M}-\mathrm{BAr}_{\mathrm{F}}-\operatorname{cod}-\mathrm{H}_{2}\right]^{+}, 30 \%\right)$; $[\alpha]_{D}^{20}=+18.7\left(\mathrm{c}=0.5, \mathrm{CHCl}_{3}\right)$. 


\section{(-)-(S)-[( $\left.\eta^{4}-1,5-c y c l o o c t a d i e n e\right)-\{4-i s o p r o p y l-2-(1-d i p h e n y l p h o s p h i n i t e-1-m e t h y l-e t h y l)-4,5-$}

\section{dihydro-oxazole \}iridium(I)]-tetrakis[3,5-bis(trifluoromethyl)phenyl]borate (Ir-8)}

Synthesis according to general procedure A using $191 \mathrm{mg}(0.537 \mathrm{mmol}) \mathbf{8}, 198 \mathrm{mg}(0.296 \mathrm{mmol}$, 0.55 equiv) [ $\operatorname{IrCl}(1,5-\mathrm{cod})]_{2}$, and $551 \mathrm{mg}$ (0.591 mmol, 1.1 equiv) $\mathrm{NaBAr}_{\mathrm{F}}$ in $10 \mathrm{~mL} \mathrm{CH}_{2} \mathrm{Cl}_{2}$.

Yield: $629 \mathrm{mg}$ (0.414 mmol, 77\%). Single crystals suitable for X-ray crystallography were obtained by recrystallisation from $\mathrm{CH}_{2} \mathrm{Cl}_{2}$ /pentane (Figure S3, p. 14).

$\mathrm{C}_{61} \mathrm{H}_{50} \mathrm{BF}_{24} \mathrm{IrNO}_{2} \mathrm{P}$ (1519.05): calcd C 48.23, $\mathrm{H}$ 3.32, $\mathrm{N}$ 0.92, O 2.11;

found C 47.99, H 3.06, N 0.93, O 2.35;

m. p. $188^{\circ} \mathrm{C}$;

${ }^{1}$ H NMR (400.1 MHz, CDCl $\left.3,300 \mathrm{~K}\right): \delta=0.03\left(\mathrm{~d},{ }^{3} \mathrm{~J}(\mathrm{H}, \mathrm{H})=6.8 \mathrm{~Hz}, 3 \mathrm{H},{ }^{i} \mathrm{Pr} \mathrm{CH}_{3}\right), 0.75\left(\mathrm{~d},{ }^{3} \mathrm{~J}(\mathrm{H}, \mathrm{H})\right.$ $\left.=6.8 \mathrm{~Hz}, 3 \mathrm{H},{ }^{i} \operatorname{Pr~CH}_{3}\right), 1.62\left(\mathrm{mc}, 1 \mathrm{H}, \operatorname{cod} \mathrm{CH}_{2}\right), 1.68\left(\mathrm{~s}, 3 \mathrm{H}, \mathrm{P}-\mathrm{O}-\mathrm{C}-\mathrm{CH}_{3}\right), 1.81\left(\mathrm{~m}, 2 \mathrm{H}, \mathrm{cod} \mathrm{CH}_{2}\right)$, 1.98 (m, 1H, cod $\mathrm{CH}_{2}$ ), 2.23 (s, 3H, P-O-C-CH $\left.\mathrm{CH}_{3}\right), 2.32$ (m, 3H, ${ }^{i} \mathrm{Pr} \mathrm{CH}$ and $\operatorname{cod}^{\mathrm{CH}_{2}}$ ), 2.55 (m, $2 \mathrm{H}$, cod $\left.\mathrm{CH}_{2}\right), 2.74(\mathrm{~m}, 1 \mathrm{H}, \operatorname{cod} \mathrm{CH}), 3.51(\mathrm{~m}, 1 \mathrm{H}, \operatorname{cod} \mathrm{CH}), 3.98\left(\mathrm{~m}, 1 \mathrm{H}\right.$, oxaz. $\left.\mathrm{CH}_{2}\right), 4.29\left(\mathrm{t},{ }^{3} \mathrm{~J}(\mathrm{H}, \mathrm{H})=\right.$ $9.9 \mathrm{~Hz}, 1 \mathrm{H}$, oxaz. $\mathrm{CH}$ ), 4.42 (dd, $J(\mathrm{H}, \mathrm{H})=9.8 \mathrm{~Hz}, 4.5 \mathrm{~Hz}, 1 \mathrm{H}$, oxaz. $\left.\mathrm{CH}_{2}\right), 4.95$ (m, 1H, cod CH), $5.17(\mathrm{~m}, 1 \mathrm{H}, \operatorname{cod} \mathrm{CH}), 7.11(\mathrm{~m}, 2 \mathrm{H}$, arom. $\mathrm{CH}), 7.39$ - 7.61 (m, 10H, arom. $\mathrm{CH}$ and $\mathrm{BAr}_{\mathrm{F}}$ para $\mathrm{CH}$ ), 7.72 (s, 8H, BAr F $_{\mathrm{F}}$ ortho $\mathrm{CH}$ ), 7.83 (m, 2H, arom. $\mathrm{CH}$ );

${ }^{31} \mathbf{P}\left\{{ }^{1} \mathbf{H}\right\}$ NMR (162.0 MHz, $\left.\mathrm{CDCl}_{3}, 300 \mathrm{~K}\right): \delta=93.4$ (s);

${ }^{19} \mathbf{F}\left\{{ }^{1} \mathbf{H}\right\}$ NMR (376.5 MHz, $\left.\mathrm{CDCl}_{3}, 300 \mathrm{~K}\right): \delta=-63.5(\mathrm{~s})$;

${ }^{13} \mathbf{C}\left\{{ }^{1} \mathbf{H}\right\}$ NMR (100.6 MHz, CDCl $\left.3,300 \mathrm{~K}\right): \delta=13.1\left({ }^{i} \mathrm{Pr} \mathrm{CH}_{3}\right), 18.7\left({ }^{i} \operatorname{Pr~CH}_{3}\right), 26.2\left(\mathrm{~d},{ }^{3} J(\mathrm{P}, \mathrm{C})=\right.$ $\left.5.8 \mathrm{~Hz}, \mathrm{P}-\mathrm{O}-\mathrm{C}-\mathrm{CH}_{3}\right), 27.0\left(\operatorname{cod} \mathrm{CH}_{2}\right), 29.7\left(\mathrm{~d}, J(\mathrm{P}, \mathrm{C})=1.9 \mathrm{~Hz}, \operatorname{cod} \mathrm{CH}_{2}\right), 32.1(\mathrm{~d}, J(\mathrm{P}, \mathrm{C})=2.3 \mathrm{~Hz}$, $\left.\operatorname{cod} \mathrm{CH}_{2}\right), 32.5\left({ }^{i} \operatorname{Pr} \mathrm{CH}\right), 33.6\left(\mathrm{~d},{ }^{3} \mathrm{~J}(\mathrm{P}, \mathrm{C})=2.7 \mathrm{~Hz}, \mathrm{P}-\mathrm{O}-\mathrm{C}-\mathrm{CH}_{3}\right), 35.9\left(\mathrm{~d}, J(\mathrm{P}, \mathrm{C})=3.5 \mathrm{~Hz}, \operatorname{cod~CH}_{2}\right)$, 61.8 (cod CH), 65.6 (cod $\mathrm{CH}$ ), 70.5 (oxaz. $\mathrm{CH}$ ), 71.0 (oxaz. $\mathrm{CH}_{2}$ ), 79.7 (d, ${ }^{2} J(\mathrm{P}, \mathrm{C})=3.8 \mathrm{~Hz}, \mathrm{P}-\mathrm{O}-\mathrm{C}-$ $\left.\mathrm{CH}_{3}\right), 97.9\left(\mathrm{~d}, J(\mathrm{P}, \mathrm{C})=12.2 \mathrm{~Hz}\right.$, cod CH), $98.9\left(\mathrm{~d}, J(\mathrm{P}, \mathrm{C})=12.6 \mathrm{~Hz}\right.$, cod CH), $117.9\left(\mathrm{~m}, \mathrm{BAr}_{\mathrm{F}}-\mathrm{C}\right)$, $125.0\left(\mathrm{q},{ }^{1} J(\mathrm{~F}, \mathrm{C})=273 \mathrm{~Hz}, \mathrm{BAr}_{\mathrm{F}} \mathrm{CF}_{3}\right), 129.0,129.3(\mathrm{~d}, J(\mathrm{P}, \mathrm{C})=11.1 \mathrm{~Hz}$, arom. $\mathrm{CH}), 129.3(\mathrm{~m}$, BAr $_{F}$ C), 129.8 (d, $J=11.9 \mathrm{~Hz}$, arom. CH), 130.2, 130.7 (d, $J(\mathrm{P}, \mathrm{C})=11.9 \mathrm{~Hz}$, arom. CH), 132.5 (d, $J(\mathrm{P}, \mathrm{C})=2.7 \mathrm{~Hz}$, arom. $\mathrm{CH}), 133.2(\mathrm{~d}, J(\mathrm{P}, \mathrm{C})=15.0 \mathrm{~Hz}$, arom. $\mathrm{CH}), 133.5(\mathrm{~d}, J(\mathrm{P}, \mathrm{C})=2.3 \mathrm{~Hz}$, arom. C), 135.2 (br s, $\left.\mathrm{BAr}_{\mathrm{F}}-\mathrm{C}\right), 161.9$ (m, BAr $\left.\mathrm{F}_{\mathrm{F}}-\mathrm{C}\right), 175.3$ (d, J(P,C) = 6.9 Hz, C=N);

IR (KBr): $\widetilde{v}=3065 \mathrm{w}, 2980 \mathrm{w}, 2957 \mathrm{w}, 2885 \mathrm{w}, 2835 \mathrm{w}, 1612 \mathrm{~m}, 1485 \mathrm{w}, 1466 \mathrm{w}, 1439 \mathrm{w}, 1381 \mathrm{w}$, 1352s, 1271s, 1161m, 1117s, 1105s, 1016m, 999m, 989m, 953m, 897m, 883m, 839m, 820 $\mathrm{w} \mathrm{cm}^{-1}$;

+FABMS, 3-NBA, m/e: = 656 ([M-BAr $]^{+}$, 100), 452 (10), 421 (8), 390 (7), 343 (5), 317 (5), 295 (6), 192 (4), 176 (4).

$[\alpha]_{D}^{20}=-31\left(\mathrm{c}=0.12, \mathrm{CHCl}_{3}\right)$. 


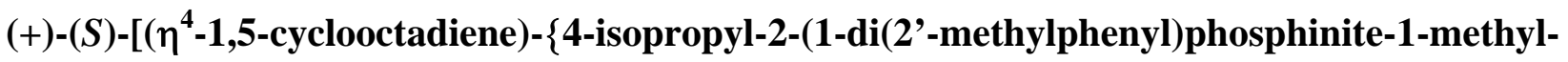
ethyl)-4,5-dihydro-oxazole\}iridium(I)]-tetrakis[3,5-bis(trifluoromethyl)phenyl]borate (Ir-9) Synthesis according to general procedure A using $97 \mathrm{mg}(0.253 \mathrm{mmol}) \mathbf{9}, 93 \mathrm{mg}(0.139 \mathrm{mmol}$, 0.55 equiv) [ $\operatorname{IrCl}(1,5 \text {-cod) }]_{2}$, and $260 \mathrm{mg}$ (0.278 mmol, 1.1 equiv) $\mathrm{NaBAr}_{\mathrm{F}}$ in $10 \mathrm{~mL} \mathrm{CH}_{2} \mathrm{Cl}_{2}$. Yield: $236 \mathrm{mg}(0.153 \mathrm{mmol}, 60 \%)$.

$\mathrm{C}_{63} \mathrm{H}_{54} \mathrm{BF}_{24} \mathrm{IrNO}_{2} \mathrm{P}$ (1547.05): calcd C 48.91, H 3.52, N 0.91, O 2.07;

found C 48.75, H 3.35, N 1.03, O 2.31;

m. p. $120^{\circ} \mathrm{C}$;

${ }^{1} \mathbf{H}$ NMR (400.1 MHz, CDCl $\left.3,300 \mathrm{~K}\right): \delta=0.26\left(\mathrm{~d},{ }^{3} \mathrm{~J}(\mathrm{H}, \mathrm{H})=6.8 \mathrm{~Hz}, 3 \mathrm{H},{ }^{i} \mathrm{Pr} \mathrm{CH}_{3}\right), 0.88\left(\mathrm{~d},{ }^{3} \mathrm{~J}(\mathrm{H}, \mathrm{H})\right.$ $\left.=6.8 \mathrm{~Hz}, 3 \mathrm{H},{ }^{i} \operatorname{Pr~CH}_{3}\right), 1.44$ (s, $\left.3 \mathrm{H},{ }^{\circ}{ }^{\mathrm{Tol}}-\mathrm{CH}_{3}\right), 1.72\left(\mathrm{mc}, 1 \mathrm{H}\right.$, cod $\left.\mathrm{CH}_{2}\right), 1.84$ (s, 3H, P-O-C-CH$)_{3}$, 1.99 - 2.67 (m, $15 \mathrm{H}$, iPr CH, P-O-C- $\mathrm{CH}_{3},{ }^{\circ} \mathrm{Tol}^{-\mathrm{CH}_{3}}$, $\operatorname{cod} \mathrm{CH}_{2}$ and $\operatorname{cod} \mathrm{CH}$ ), 3.26 (m, $1 \mathrm{H}$, cod $\mathrm{CH}$ ), $4.02\left(\mathrm{~m}, 1 \mathrm{H}\right.$, oxaz. $\left.\mathrm{CH}_{2}\right), 4.23\left(\mathrm{t},{ }^{3} \mathrm{~J}(\mathrm{H}, \mathrm{H})=9.3 \mathrm{~Hz}, 1 \mathrm{H}\right.$, oxaz. $\left.\mathrm{CH}\right), 4.42(\mathrm{dd}, J(\mathrm{H}, \mathrm{H})=9.9 \mathrm{~Hz}, 3.8$ $\mathrm{Hz}, 1 \mathrm{H}$, oxaz. $\left.\mathrm{CH}_{2}\right), 4.77(\mathrm{~m}, 1 \mathrm{H}$, cod $\mathrm{CH}), 5.28(\mathrm{~m}, 1 \mathrm{H}$, cod $\mathrm{CH}), 7.13$ (m, 2H, arom. $\left.\mathrm{CH}\right), 7.29-$ 7.41 (m, 4H, arom. CH), 7.52 (m, 6H, arom. $\mathrm{CH}$ and $\mathrm{BAr}_{\mathrm{F}}$ para $\mathrm{CH}$ ), 7.72 (s, 8H, $\mathrm{BAr}_{\mathrm{F}}$ ortho $\mathrm{CH}$ ), 8.44 (m, $1 \mathrm{H}$, arom. $\mathrm{CH})$;

${ }^{31} \mathbf{P}\left\{{ }^{1} \mathbf{H}\right\}$ NMR (162.0 MHz, $\left.\mathrm{CDCl}_{3}, 300 \mathrm{~K}\right): \delta=106.1$ (s);

${ }^{19} \mathbf{F}\left\{{ }^{1} \mathbf{H}\right\}$ NMR (376.5 MHz, $\left.\mathrm{CDCl}_{3}, 300 \mathrm{~K}\right): \delta=-63.5$ (s);

${ }^{13} \mathbf{C}\left\{{ }^{1} \mathbf{H}\right\}$ NMR (100.6 MHz, CDCl $\left.3,300 \mathrm{~K}\right): \delta=13.7\left({ }^{i} \operatorname{Pr~CH}_{3}\right), 19.0\left({ }^{i} \operatorname{Pr~CH}_{3}\right), 23.1\left({ }^{\circ} \mathrm{Tol} \mathrm{CH}_{3}\right)$, $23.2\left({ }^{\circ} \mathrm{Tol} \mathrm{CH} 3\right), 26.2\left(\mathrm{P}-\mathrm{O}-\mathrm{C}-\mathrm{CH}_{3}\right), 27.3\left(\operatorname{cod} \mathrm{CH}_{2}\right), 28.4\left(\mathrm{cod} \mathrm{CH}_{2}\right), 31.0\left(\mathrm{cod} \mathrm{CH}_{2}\right), 32.8\left({ }^{i} \mathrm{Pr} \mathrm{CH}\right)$, $32.9\left(\mathrm{~d},{ }^{3} \mathrm{~J}(\mathrm{P}, \mathrm{C})=2.7 \mathrm{~Hz}, \mathrm{P}-\mathrm{O}-\mathrm{C}-\mathrm{CH}_{3}\right), 36.5\left(\operatorname{cod} \mathrm{CH}_{2}\right), 63.7(\operatorname{cod} \mathrm{CH}), 65.4(\operatorname{cod} \mathrm{CH}), 70.1$ (oxaz. $\mathrm{CH}_{2}$ ), 71.1 (oxaz. $\mathrm{CH}$ ), 80.4 (d, $\left.{ }^{2} J(\mathrm{P}, \mathrm{C})=6.5 \mathrm{~Hz}, \mathrm{P}-\mathrm{O}-\mathrm{C}-\mathrm{CH}_{3}\right), 95.8$ (d, $J(\mathrm{P}, \mathrm{C})=15.7 \mathrm{~Hz}, \operatorname{cod} \mathrm{CH}$ ), $99.2(\mathrm{~d}, J(\mathrm{P}, \mathrm{C})=12.6 \mathrm{~Hz}$, cod $\mathrm{CH}), 117.9\left(\mathrm{~m}, \mathrm{BAr}_{\mathrm{F}}-\mathrm{C}\right), 125.0\left(\mathrm{q},{ }^{1} \mathrm{~J}(\mathrm{~F}, \mathrm{C})=273 \mathrm{~Hz}, \mathrm{BAr}_{\mathrm{F}} \mathrm{CF}_{3}\right)$, 129.0, $129.3(\mathrm{~m}, \mathrm{BAr} F \mathrm{C}), 129.4(\mathrm{~d}, J(\mathrm{P}, \mathrm{C})=12.8 \mathrm{~Hz}$, arom. $\mathrm{CH}), 132.8,133.1,133.9,135.2$ (br s, $\left.\mathrm{BAr}_{\mathrm{F}}-\mathrm{C}\right), 141.6,143.2,161.9\left(\mathrm{~m}, \mathrm{BAr}_{\mathrm{F}}-\mathrm{C}\right), 173.3(\mathrm{C}=\mathrm{N})$;

IR (KBr): $\tilde{v}=2980 \mathrm{w}, 2889 \mathrm{w}, 2841 \mathrm{w}, 1612 \mathrm{~m}, 1483 \mathrm{w}, 1468 \mathrm{w}, 1452 \mathrm{w}, 1352 \mathrm{~s}, 1273 \mathrm{~s}, 1161 \mathrm{~m}$, 1121s, 1016m, 1005m, 989m, 955w, 897m, 885m, 839m, 806 $\mathrm{w} \mathrm{cm}^{-1}$;

+FABMS, 3-NBA, m/e: 684 ([M-BAr $]^{+}, 100$ ), 574 (19), 477(5), 435 (5), 419 (12), 387 (5), 329 (12), 311 (6), 295 (5), 192 (5), 176 (4), 115 (6), 105 (5).

$[\alpha]_{D}^{20}=+22\left(\mathrm{c}=0.11, \mathrm{CHCl}_{3}\right)$. 


\section{Single Crystal X-ray Structures}

Single crystals of Ir-6, Ir-8, and Ir-12 were obtained from $\mathrm{Et}_{2} \mathrm{O}$ or dichloromethane, by layering solutions with the non-solvent pentane. The crystals were mounted at random orientation on a glass fiber and the data were collected on a KappaCCD four-circle diffractometer at $173 \mathrm{~K}$. The space group was determined from the systematic absences using the software Collect suite (Nonius BV, 2002). Integration of frames was done with EvalCCD (Bruker Nonius BV, 2002).

The structures were solved by direct methods with SIR92 or SIR97 ${ }^{[8]}$ and refined with CRYSTALS ${ }^{[9]}$ by Fourier methods by full matrix least squares by minimizing the function $\left[\Sigma w\left(F_{0}-\right.\right.$ $\left.\left.(1 / k) F_{c}\right)^{2}\right]$ and using anisotropic displacement parameters for all atoms. No extinction correction was deemed necessary. A Chebychev polynomial was applied as a weighting scheme. ${ }^{[10]}$ Restraints were applied to disordered $\mathrm{CF}_{3}$ groups of $\mathrm{BAr}_{\mathrm{F}}{ }^{-}$anions.

The contribution of the hydrogen atoms, in their calculated position, $(\mathrm{C}-\mathrm{H}=0.95 \AA$, $\left.B(H)=1.3 / 1.5\left[B\left(C_{\text {bonded }}\right)\right]\left(\AA^{2}\right)\right)$, was included in the refinement using a riding model. Refining Flack's parameter ${ }^{[11]}$ tested the handedness of the structures. Upon convergence, the final Fourier difference map showed no significant peaks. The scattering factors used, corrected for the real and imaginary parts of the anomalous dispersion, were taken from the literature. ${ }^{[12]}$ For the generation of graphics, ORTEP-3 for Windows Version 1.073 was used. ${ }^{[13]}$

Crystal and structure refinement data for solid state structures presented in this thesis are presented in the following tables. CIF files can be obtained from the Cambridge Crystallographic Data Centre using deposition numbers 201708 (Ir-6), 224190 (Ir-8), and 201702 (Ir-12). Copies of the data can be obtained, free of charge, on application to the CCDC, 12 Union Road, Cambridge CB2 1EZ, UK [fax: +44 (1223) 336 033; e-mail: deposit@ccdc.cam.ac.uk]. 


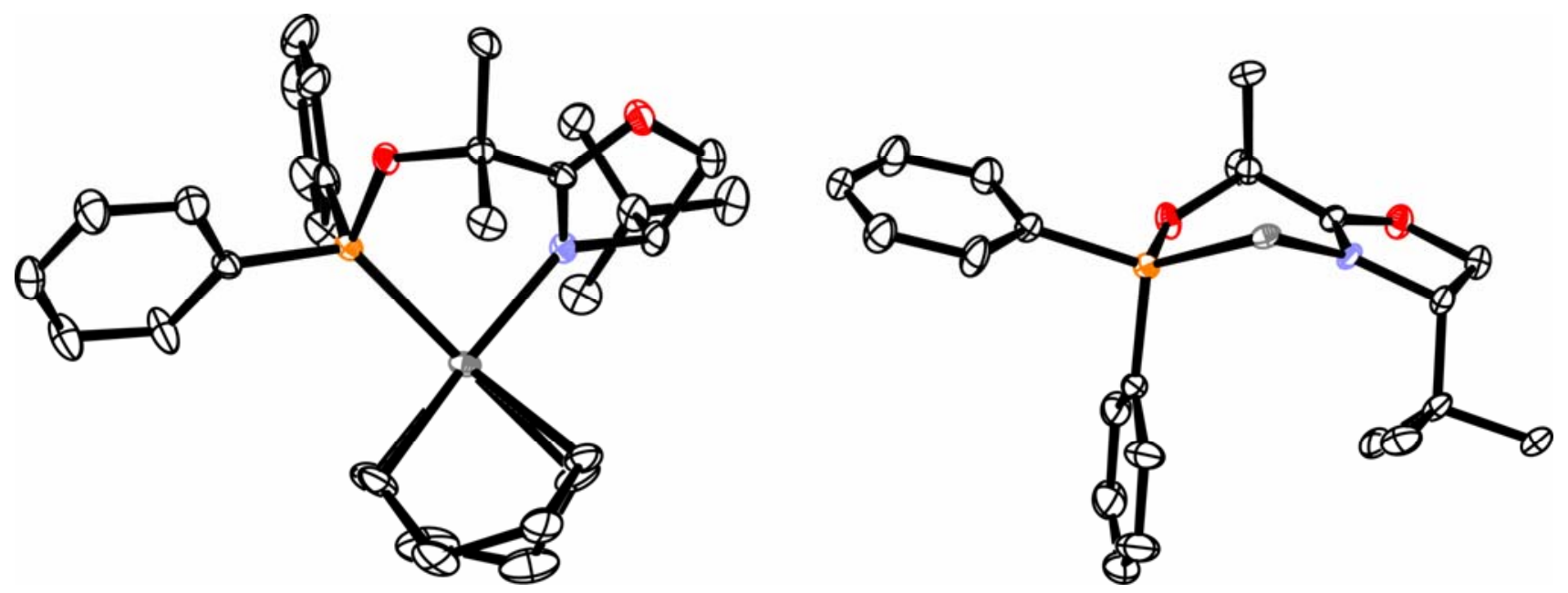

Figure S1: Two views of the cation of Ir-6, anion $\mathrm{BAr}_{\mathrm{F}}{ }^{-}$, hydrogen atoms, and on the right hand side also cod are omitted for clarity, ellipsoids drawn at 30\% probability with ORTEP-3. ${ }^{[13]}$
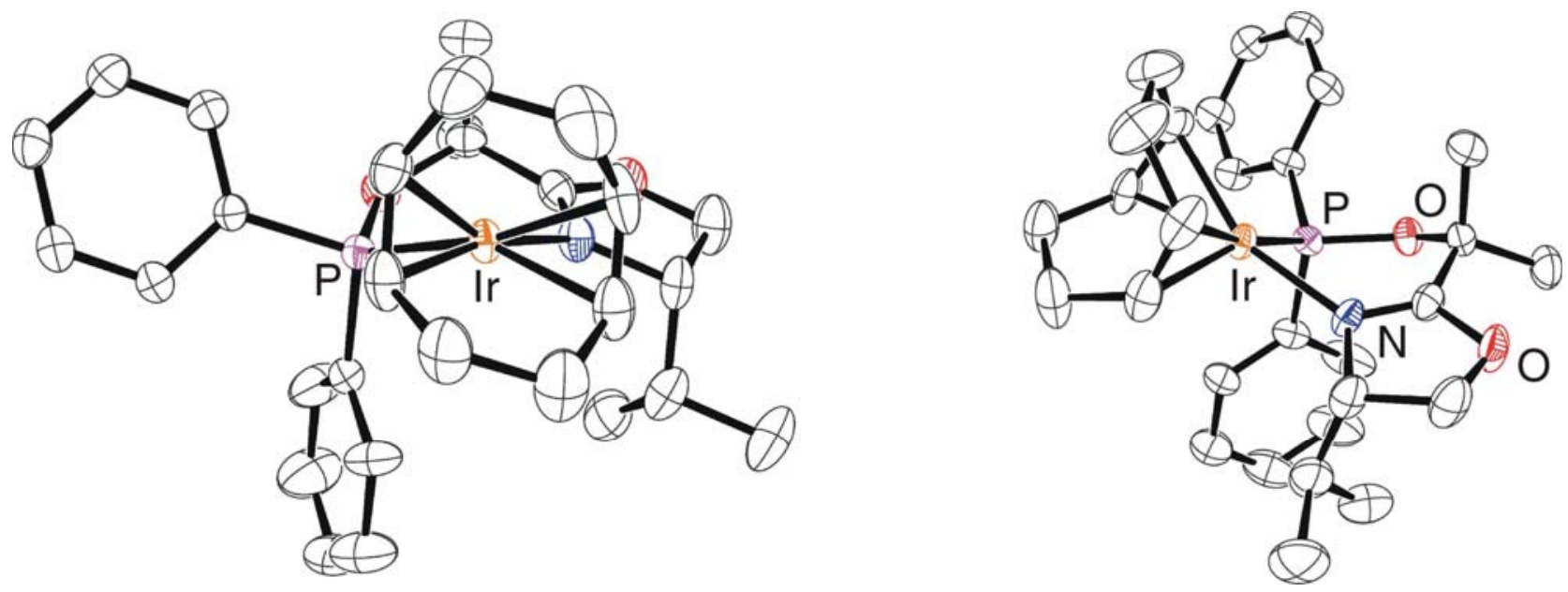

Figure S2: X-ray crystal structure of Ir-8, anion $\mathrm{BAr}_{\mathrm{F}}^{-}$and hydrogen atoms are omitted for clarity, ellipsoids drawn at 40\% probability with ORTEP-3. ${ }^{[13]}$ 

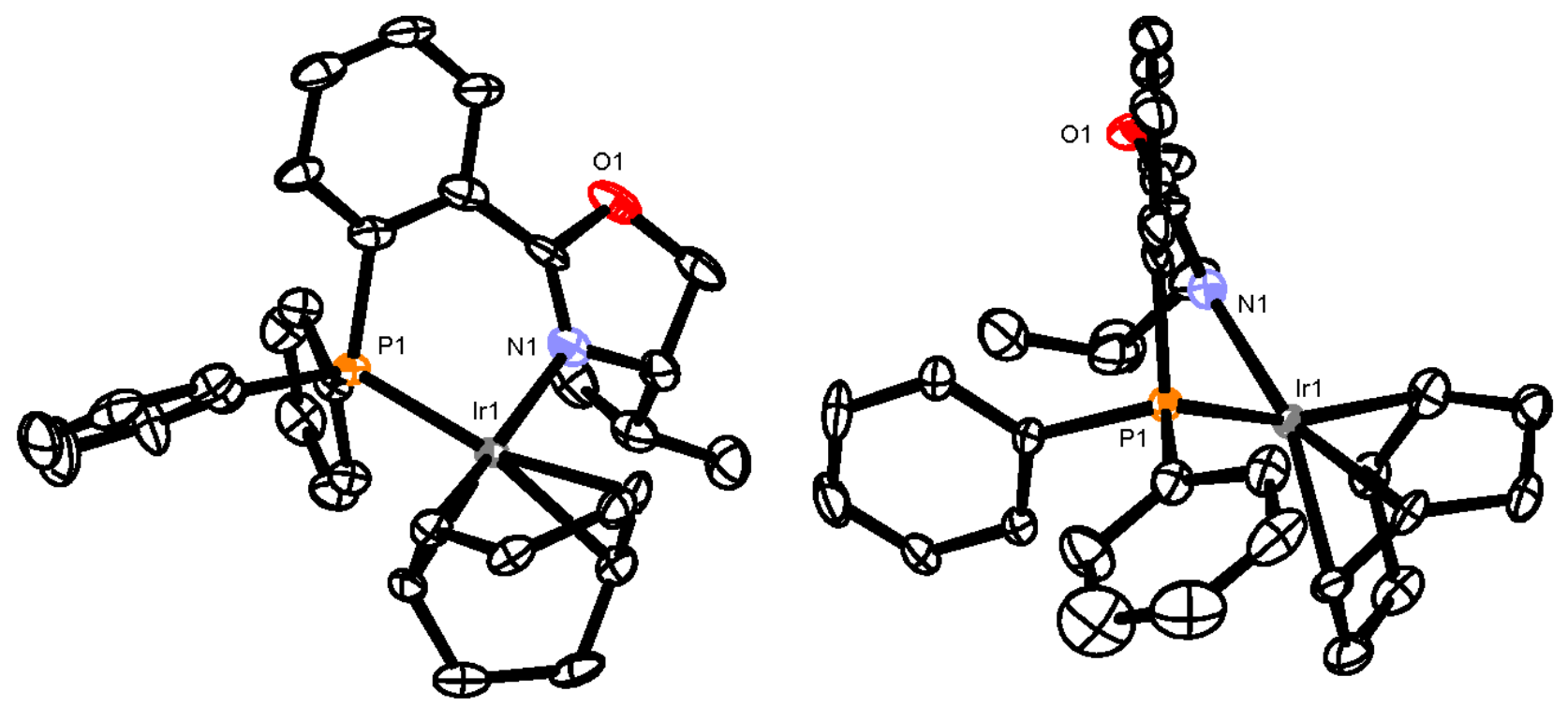

Figure S3: Two views of the cation of Ir-12, anion $\mathrm{BAr}_{\mathrm{F}}^{-}$and hydrogen atoms are omitted for clarity, ellipsoids drawn at 30\% probability with ORTEP-3. ${ }^{[13]}$

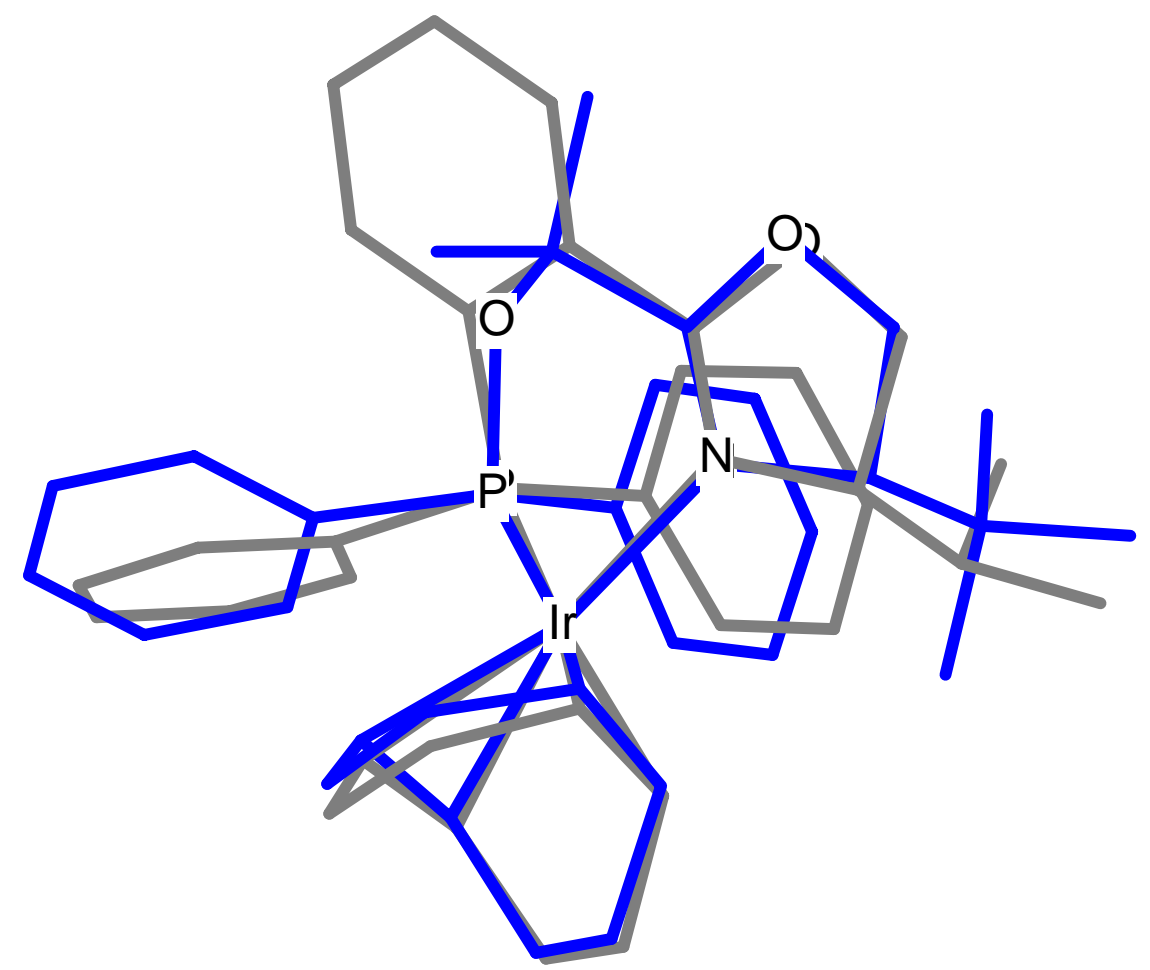

Figure S4: Superposition of solid-state structures: Ir-6 (blue) with standard iridium-PHOX complex Ir-12 (gray). 
Table S1: Crystal data and structure refinement of iridium phosphinite complexes Ir-6 and Ir-8.

\begin{tabular}{|c|c|c|}
\hline compound no. & Ir-6 & Ir-8 \\
\hline empirical formula & $\mathrm{C}_{30} \mathrm{H}_{40} \mathrm{IrNO}_{2} \mathrm{P} \cdot \mathrm{C}_{32} \mathrm{H}_{12} \mathrm{BF}_{24}$ & $\mathrm{C}_{29} \mathrm{H}_{38} \mathrm{IrNO}_{2} \mathrm{P} \cdot \mathrm{C}_{32} \mathrm{H}_{12} \mathrm{BF}_{24} \cdot 0.5 \mathrm{CHCl}_{3}$ \\
\hline formula weight $\left[\mathrm{g} \mathrm{mol}^{-1}\right]$ & 1533.06 & 1519.05 \\
\hline diffractometer & KappaCCD & KappaCCD \\
\hline radiation & Mo K $\alpha$, graphite monochr. & 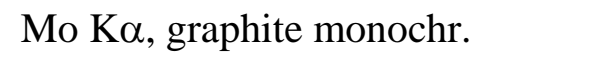 \\
\hline wavelength $[\AA]$ & 0.71073 & 0.71073 \\
\hline temperature $[\mathrm{K}]$ & 173 & 173 \\
\hline crystal system & triclinic & triclinic \\
\hline space group (no.) & $P 1(1)$ & $P 1(1)$ \\
\hline \multirow[t]{6}{*}{ unit cell dimensions $\left[\AA ;^{\circ}\right]$} & $\mathrm{a}=12.8996(3)$ & $\mathrm{a}=12.7425(1) ; \alpha=87.9035(5)$ \\
\hline & $\alpha=105.044(1)$ & \\
\hline & $\mathrm{b}=12.9357(1)$ & $\mathrm{b}=12.9920(1) ; \beta=74.6505(5)$ \\
\hline & $\beta=92.7318(17)$ & \\
\hline & $\mathrm{c}=22.1831(3)$ & $c=23.0228(2)$ \\
\hline & $\gamma=117.3655(13)$ & $\gamma=61.9161(5)$ \\
\hline unit cell volume $\left[\AA^{3}\right]$ & 3113.9 & 3225.3 \\
\hline$Z$ & 2 & 2 \\
\hline calculated density $\left[\mathrm{g} \mathrm{cm}^{-3}\right]$ & 1.635 & 1.626 \\
\hline absorption coeff. $\mu$ [mm $\left.{ }^{-1}\right]$ & 2.285 & 2.269 \\
\hline $\mathrm{F}(000)$ & 1517.026 & 1559.600 \\
\hline crystal size & $0.29 \times 0.20 \times 0.09 \mathrm{~mm}$ & $0.26 \times 0.20 \times 0.20 \mathrm{~mm}$ \\
\hline absorption correction & none & multi-scan \\
\hline$\theta$ range for data collection & $4.08^{\circ}$ to $32.00^{\circ}$ & $4.60^{\circ}$ to $30.06^{\circ}$ \\
\hline \multirow[t]{3}{*}{ index ranges } & $-19 \leq \mathrm{h} \leq 19$ & $-17 \leq \mathrm{h} \leq 17$ \\
\hline & $-19 \leq \mathrm{k} \leq 19$ & $-18 \leq \mathrm{k} \leq 16$ \\
\hline & $-33 \leq 1 \leq 33$ & $-32 \leq 1 \leq 32$ \\
\hline reflections collected & 112109 & 32944 \\
\hline unique reflections & $37354[\mathrm{R}$ (int) $=0.08]$ & $25383[\mathrm{R}(\mathrm{int})=0.00]$ \\
\hline data / parameters & $28695 / 1740$ & $25383 / 1873$ \\
\hline goodness-of-fit & 1.0325 & 1.0578 \\
\hline $\mathrm{R} 1[\mathrm{I}>2 \sigma(\mathrm{I})]$ & 0.0429 & 0.0312 \\
\hline wR2 [I>2 $\sigma(I)]$ & 0.0462 & 0.0355 \\
\hline Flack $x$ & $0.010(4)$ & $0.017(3)$ \\
\hline largest diff. peak $\left[\mathrm{e}^{-} \AA^{-3}\right]$ & 3.85 & 1.36 \\
\hline largest diff. hole $\left[\mathrm{e}^{-} \AA^{-3}\right]$ & -2.43 & -1.33 \\
\hline CCDC deposition no. & 201708 & 224190 \\
\hline
\end{tabular}


Table S2: Crystal data and structure refinement of iridium PHOX complex Ir-12.

\begin{tabular}{|c|c|}
\hline compound no. & Ir-12 \\
\hline empirical formula & $\mathrm{C}_{32} \mathrm{H}_{36} \mathrm{IrNOP} \cdot \mathrm{C}_{32} \mathrm{H}_{12} \mathrm{BF}_{24}$ \\
\hline formula weight $\left[\mathrm{g} \mathrm{mol}^{-1}\right.$ ] & 1537.05 \\
\hline diffractometer & KappaCCD \\
\hline radiation & Mo Ka, graphite monochr. \\
\hline wavelength [A] & 0.71073 \\
\hline temperature $[\mathrm{K}]$ & 193 \\
\hline crystal system & monoclinic \\
\hline space group (no.) & $P 2_{1}(4)$ \\
\hline \multirow[t]{4}{*}{ unit cell dimensions $\left[\AA ̊ ;^{\circ}\right]$} & $\mathrm{a}=21.1948(5)$ \\
\hline & $\mathrm{b}=13.6660(2)$ \\
\hline & $\beta=108.9886(17)$ \\
\hline & $\mathrm{c}=22.8714(4)$ \\
\hline unit cell volume $\left[\AA^{3}\right]$ & 6264.2 \\
\hline$Z$ & 4 \\
\hline calculated density $\left[\mathrm{g} \mathrm{cm}^{-3}\right]$ & 1.630 \\
\hline absorption coeff. $\mu\left[\mathrm{mm}^{-1}\right]$ & 2.271 \\
\hline $\mathrm{F}(000)$ & 3034.033 \\
\hline crystal size & $0.32 \times 0.26 \times 0.22 \mathrm{~mm}$ \\
\hline absorption correction & none \\
\hline$\theta$ range for data collection & $4.11^{\circ}$ to $30.00^{\circ}$ \\
\hline \multirow[t]{3}{*}{ index ranges } & $-29 \leq \mathrm{h} \leq 29$ \\
\hline & $-19 \leq \mathrm{k} \leq 19$ \\
\hline & $-32 \leq \mathrm{l} \leq 22$ \\
\hline reflections collected & 75464 \\
\hline unique reflections & 34313 [R(int) = 0.07] \\
\hline data / parameters & $24578 / 1802$ \\
\hline goodness-of-fit & 1.0441 \\
\hline $\mathrm{R} 1[\mathrm{I}>2 \sigma(\mathrm{I})]$ & 0.0594 \\
\hline $\mathrm{wR} 2[\mathrm{I}>2 \sigma(\mathrm{I})]$ & 0.0678 \\
\hline Flack $x$ & --- \\
\hline largest diff. peak $\left[\mathrm{e}^{-} \AA^{-3}\right]$ & 3.82 \\
\hline largest diff. hole $\left[\mathrm{e}^{-} \AA^{-3}\right]$ & -3.52 \\
\hline CCDC deposition no. & 201702 \\
\hline
\end{tabular}




\section{References}

${ }^{[1]}$ Still, W. C.; Kahn, M.; Mitra, A. J. Org. Chem. 1978, 43, 2923-2925.

[2] Perrin, D. D.; Armarego, W. L. F. Purification of Laboratory Chemicals, ButterworthHeinemann, Oxford, 1988.

${ }^{[3]}$ Schnider, P. Ph.D. dissertation, University of Basel 1995.

${ }^{[4]}$ Hilgraf, R. Ph.D. dissertation, University of Basel 2000.

[5] (a) Allen, J. V.; Williams, J. M. J. Tetrahedron: Asymmetry 1994, 5, 277-282; (b) Pridgen, L. N.; Miller, G. J. Heterocyclic Chem. 1983, 20, 1223-1230.

${ }^{[6]}$ Blackmond, D. G.; Lightfoot, A.; Pfaltz, A.; Rosner, T.; Schnider, P.; Zimmermann, N. Chirality 2000, 12, 442-449.

[7] (a) Taube, R.; Wache, S. J. Organomet. Chem. 1992, 428, 431-442; (b) Reger, D. L.; Wright, T. D.; Little, C. A.; Lamba, J. J. S.; Smith, M. D. Inorg. Chem. 2001, 40, 3810-3814. (c) see also: Leaser, Jr., J. L.; Cvetovich, R.; Tsay, F.-R.; Dolling, U.; Vickery, T.; Bachert, D. J. Org. Chem. 2003, 68, 3695-3698.

[8] (a) SIR92: Altomare, A.; Cascarano, G.; Giacovazzo, C.; Guagliardi, A. J. Appl. Crystallogr. 1993, 26, 343-350; (b) SIR97: Altomare, A.; Burla, M. C.; Camalli, M.; Cascarano, G. L.; Giacovazzo, C.; Guagliardi, A.; Moliterni, A. G. G.; Polidori, G.; Spagna, R. J. Appl. Crystallogr. 1999, 32, 115-119.

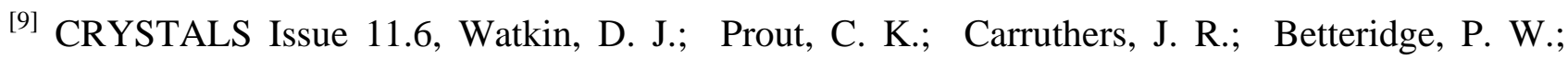
Cooper, R. I. Chemical Crystallography Laboratory, University of Oxford, Oxford, 2001.

${ }^{[10]}$ Carruthers, J. R.; Watkin, D. J. Acta Crystallogr., Sect. A 1979, 35, 698-699.

${ }^{[11]}$ Flack, H. D. Acta Crystallogr., Sect. A 1983, A 39, 876-881.

[12] International Tables for X-ray Crystallography; Wilson, A. J. C. Ed.; Kluwer Academic Publisher, Dordrecht, The Netherlands, 1992; Vol. C.

${ }^{[13]}$ Farrugia, L. J. J. Appl. Crystallogr. 1997, 30, 565. 